\title{
Study of gamma-ray background noise for radioactive waste drum characterization with plastic scintillators
}

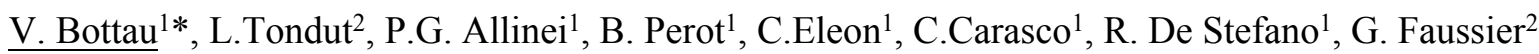 \\ ${ }^{1}$ CEA, DEN, Cadarache, DTN, SMTA, Nuclear Measurement Laboratory \\ ${ }^{2}$ Orano Business Unit Recycling \\ *vincent.bottau@cea.fr
}

\begin{abstract}
In the framework of the radioactive waste drum characterization using neutron coincidence counting, the Nuclear Measurement Laboratory of CEA Cadarache is studying plastic scintillators as an alternative to ideal but costly ${ }^{3} \mathrm{He}$ gas proportional counters. Plastic scintillators are at least 5 times cheaper for the same detection efficiency, and in addition, they detect fast neutrons about three orders of magnitude faster than

${ }^{3}$ He detectors. However, they are sensitive to gamma rays, which implies the necessity to identify precisely gamma background sources that may affect the useful signal. This paper presents a detailed analysis of the gamma-ray spectrum of a radioactive waste drum containing glove box filters contaminated by plutonium dioxide. Gamma emissions accompanying inelastic scattering $\left(n, n^{\prime}\right)$ and $(\alpha, n)$ reactions that can lead to neutrongamma coincidences parasitizing useful coincidences from plutonium spontaneous fissions are identified. Some of these parasitic gamma rays having energies up to several $\mathrm{MeV}$, we plan to reject high-energy scintillator pulses with an electronics rejection threshold above $1 \mathrm{MeV}$, which should preserve the major part of useful fission neutron pulses.
\end{abstract}

\section{INTRODUCTION}

In the context of passive neutron measurements for nuclear material characterization, conventional coincidence collars based on helium 3 gas counters permits a temporal discrimination of spontaneous fissions neutrons and parasitic neutrons from $(\alpha, n)$ reactions. ${ }^{3} \mathrm{He}$ proportional counters are the golden standard because they show a very high capture cross section for thermal neutrons, leading to high detection efficiency when surrounding detectors with polyethylene moderator, while being practically insensitive to gamma rays. However, the global shortage on ${ }^{3} \mathrm{He}$ gas [1] has greatly increased their cost in the past 15 years, leading to worldwide initiatives to look for cheaper alternatives with equivalent performances [2]. The Nuclear Measurements Laboratory of CEA, DEN, Cadarache, is currently studying the use of lowcost Polyvinyl Toluene (PVT) plastic scintillators as a potential alternative [3] [4] that have the advantage of being sensitive to fast neutrons, thus avoiding thermalizing materials and offering a nanosecond response time. Consequently, very short coincidence windows of a few tens of nanoseconds allow greatly reducing accidental coincidences compared to three order of magnitude slower ${ }^{3} \mathrm{He}$ detection systems.

However, plastic scintillators are very sensitive to gamma rays. Some of them are emitted in coincidence with $(\alpha, n)$ neutrons often encountered with plutonium oxides and alpha bearing waste, leading to neutron-gamma coincidences that penalize the detection of plutonium spontaneous fission coincidences. Other correlated gamma emissions may also occur in neutron-induced reactions, such as (n,n') inelastic scattering. Pulse Shape Discrimination (PSD) liquid organic scintillators are commonly used to parry gamma sensitivity, but they would not constitute a cost-effective alternative to ${ }^{3} \mathrm{He}$ counters for radioactive waste package characterization. Indeed, large detection volumes are needed to preserve coincidence counting statistics and therefore, a large number of PSD scintillators should be implemented as their size is limited. Actually, the difference between neutron and gamma ray pulse tails is not preserved in large PSD scintillators due to light multiple scattering [5] [6]. Finally, a large array of small (maximum 5 inches) PSD scintillators would increase the cost to a similar level as ${ }^{3} \mathrm{He}$ detectors, not to mention flammability and toxicity of organic liquid scintillators. In addition, PSD information lies in pulse tails and would be damaged by pulse pile-up in case of high count rate, as it is the case with some radioactive waste.

In view to find mitigations to gamma sensitivity of plastic scintillators, this paper reports the gamma spectrum analysis of a $120 \mathrm{~L}$ waste drum containing glove box filters contaminated by plutonium dioxide powder with a focus on gamma rays emitted by $(\alpha, x),\left(n, n^{\prime}\right)$ and $(n, \gamma)$ reactions.

\section{GAMMA SPECTRUM}

The High Purity Germanium (HPGe) detector used for the measurement is a planar Broad Energy Germanium (BEGe) from Mirion Technologies (Canberra), equipped with a CP5 Cryo-Pulse. It was in quasi-contact with a $120 \mathrm{~L}$ radioactive waste drum containing glove box filters contaminated with plutonium dioxide $\left(\mathrm{PuO}_{2}\right)$.

A $1 \mathrm{~mm}$ tin plate was placed in front of the detector to clean very low energy gamma rays from the spectrum, especially the very intense $59.5 \mathrm{keV}$ line of ${ }^{241} \mathrm{Am}$. The acquisition electronics is a DSALX from Mirion-Canberra, including high-voltage, amplification, pulse digitization and processing, and gamma spectrum storage. Data acquisition and processing is performed with Genie2000 gamma spectrometry software from Mirion-Canberra. The measurement lasted over $53 \mathrm{~h}$, 
from which $38,9 \mathrm{~h}$ of real acquisition time with a dead time of $27 \%$. Registered pulses are coded on 16384 channels and the resulting spectrum recorded on the $[0-6400] \mathrm{keV}$ range is given in Figure 1.

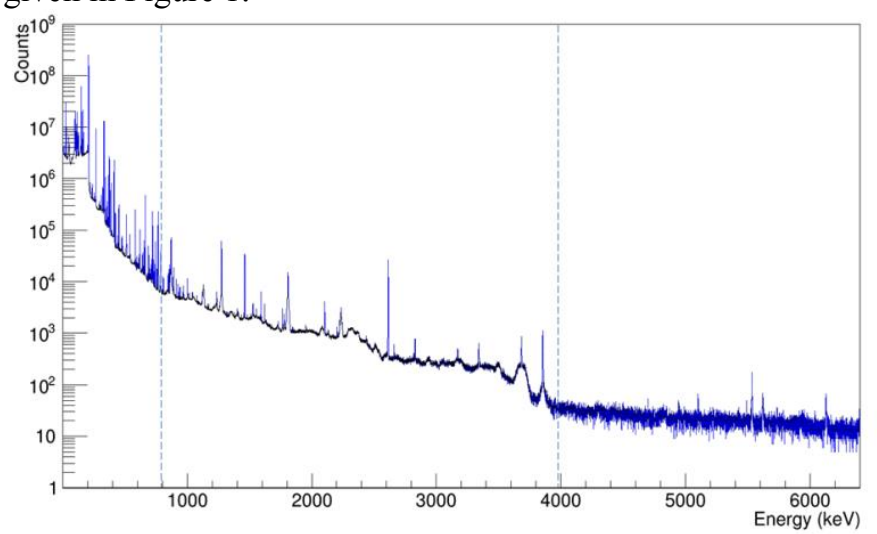

Figure 1 : Gamma spectrum of the $120 \mathrm{~L}$ plutonium waste drum resulting from a 38.9 hours real time measurement with a BEGe planar HPGe. Three main regions separated by dotted lines (from 0 to $800 \mathrm{keV}, 800$ to $4000 \mathrm{keV}$ and above $4000 \mathrm{keV}$ ) are analysed

Three main regions of interest are identified.

- The region below $800 \mathrm{keV}$ includes classical gamma rays following americium and plutonium decays. Note that the lowenergy threshold setting will be discussed in section III, in particular to cut the residual $59.5 \mathrm{keV}$ peak.

- The region between $800 \mathrm{keV}$ and $4000 \mathrm{keV}$ includes gamma rays emitted by natural radionuclides $\left({ }^{40} \mathrm{~K}\right.$, thorium chain), $(\alpha, x)$ reactions on light nuclei, and $\left(n, n^{\prime}\right)$ inelastic scattering reactions. This region represents the very heart of this work, because reaction gamma rays emitted in correlation with a neutron can lead to real but parasitic neutron-gamma coincidence, which are indistinguishable from useful spontaneous fission coincidences. It corresponds also to the energy range in which the high-energy threshold will be adjusted as discussed in section III. Expansions of the 800$1800 \mathrm{keV}$ and of the $1800-4000 \mathrm{keV}$ regions are shown in Figure 2 and Figure 3, respectively, showing a large number of peaks, some of which broadened by Doppler effect due to the short life time of the reaction product excited state. This excited nucleus is indeed still slowing down when the deexcitation gamma ray is emitted.

- a region between $4000 \mathrm{keV}$ and $6400 \mathrm{keV}$ with few peaks due to neutron inelastic scattering, which can also generate parasitic neutron-gamma coincidence. The high-energy region of the spectrum reported in Figure 4 shows neutron-induced radiative capture and inelastic scattering gamma rays on carbon and oxygen nuclei present inside the waste drum. The artefact peak near $5500 \mathrm{keV}$ is due to DSA-LX electronics. This peak is also present during background noise acquisitions. The $4946.31 \mathrm{keV}$ radiative capture gamma ray on ${ }^{12} \mathrm{C}$ nuclei is weak but present, as well as the $6128.63 \mathrm{keV}$ inelastic scattering gamma ray on ${ }^{16} \mathrm{O}$ accompanied by its single escape (SE) and double escape (DE) peaks. The ${ }^{16} \mathrm{O}(\mathrm{n}, \mathrm{n}$ ') reaction may also lead to $6917 \mathrm{keV}$ and $7117 \mathrm{keV}$ gamma rays [7] but they are beyond the $6400 \mathrm{keV}$ end-energy of the spectrum.

\section{THRESHOLDS SETTINGS FOR PLASTIC SCINTILLATORS}

In view to limit parasitic non-fission coincidences in plutonium measurements with plastic scintillators, we plan to use low- and high-energy thresholds to cut as many gamma rays as possible. Uncorrelated gamma rays from alpha and beta disintegrations, and from $(\alpha, \mathrm{x})$ reactions do not create coincidences, except from cross talk between close detectors but such events can be discarded by time rejection as explained in [3]. However, as mentioned above, prompt gamma rays accompanying $(\alpha, n)$ and neutron inelastic scattering reactions, as well as cascade gamma rays following disintegrations or reactions, can create non-fission coincidences. In order to find a trade-off between neutron preservation and gamma-ray elimination using low- and highenergy cutoffs, is it important to take into account the difference in scintillation light output between neutron and gamma detection in plastic scintillators. Indeed, the quenching effect reduces the light output generated by heavy particles, such as recoil protons after neutron elastic scattering on hydrogen nuclei, compared to the same energy deposition by a gamma ray [8]. The energy deposition of a proton is therefore converted in $\mathrm{MeV}$ equivalent electron (MeVee), which is the energy that an electron would release by slowing down in the plastic scintillator to produce the same light pulse. A previous study concerning BC-420 plastic scintillators [9] gives the following conversion in case for recoil protons:

$$
\mathrm{E}(\mathrm{MeVee})=0.0364 \mathrm{E}_{\mathrm{n}}^{2}+0.125 \mathrm{E}_{\mathrm{n}}
$$

and in case of recoil carbon nuclei [5] [10] the relationship is:

$$
\mathrm{E}(\mathrm{MeVee})=0.02 \mathrm{E}_{\mathrm{n}}
$$

where $E_{n}$ is the energy deposited by the neutron, in $\mathrm{MeV}$.

The only consider here fast neutron reactions inducing recoil protons in plastic scintillators.

Figure 5 shows a Maxwell spectrum representing the energy distribution of neutrons emitted by spontaneous fission of ${ }^{240} \mathrm{Pu}$, which is the major spontaneous fission emitter [11]:

$$
N(E)=\sqrt{E} \exp (-E / 1.32 \mathrm{MeV})
$$

Table 1 reports fission neutron losses when applying different low- and high-energy thresholds. It is important to remind that neutron elastic scattering leads to uniform energy deposition between 0 and $E_{n}$ (incident neutron energy) on hydrogen nuclei in plastic scintillators. Consequently, neutron detection is still possible when $E_{n}$ is larger than the high-energy threshold, in case of energy deposition smaller than this highenergy threshold. On the other hand, a neutron with $\mathrm{E}_{\mathrm{n}}$ larger than the low-energy threshold can be discarded if its energy deposition is below this low-energy threshold. The same is true for partial photon energy deposit in plastic scintillators, in which Compton scattering is the major interaction process. Therefore, Table 1 just provides a qualitative idea of neutron losses. The real thresholds will be determined experimentally based on signal-to-noise ratio (i.e. spontaneous fission vs. parasite coincidences using ${ }^{252} \mathrm{Cf}$ and $\mathrm{AmBe}$ sources, respectively) and detection limit considerations (using $\mathrm{Pu}$ samples).

Following the gamma spectrum analysis, three low-energy thresholds are considered

- a $60 \mathrm{keVee}$ threshold allows cutting the contribution of the 
intense $59.54 \mathrm{keV}$ gamma ray of ${ }^{241} \mathrm{Am}$ in plastic scintillators. In fact, this gamma ray mainly deposits a much lower energy than $59 \mathrm{keV}$ by Compton scattering. The $60 \mathrm{keVee}$ threshold would cut energy depositions below $426.9 \mathrm{keV}$ for neutrons, which leads to about $4 \%$ neutron losses of the Maxwell spectrum;

- a $130 \mathrm{keVee}$ threshold would cut the intense gamma and Xray signal from americium and plutonium disintegrations but also neutron energy depositions up to $836.3 \mathrm{keV}$, i.e. $11 \%$ of spontaneous fission neutrons;
- a $210 \mathrm{keVee}$ threshold would cut the intense ${ }^{237} \mathrm{U}$ and plutonium gamma rays, especially the $208 \mathrm{keV}$ peak of ${ }^{241} \mathrm{Am} /{ }^{237} \mathrm{U}$, which is the most intense line of the whole gamma spectrum, and its associated Compton continuum. In fact, the $208 \mathrm{keV}$ peak Compton edge is close to $93 \mathrm{keV}$ and even if PVT scintillators have a poor energy resolution, $208 \mathrm{keV}$ gamma rays can easily be eliminated even with the previous $130 \mathrm{keV}$ threshold. Just for information, the $208 \mathrm{keV}$ threshold would cut neutron energy depositions up to $1236 \mathrm{keV}$, i.e. $20 \%$ of spontaneous fission neutrons

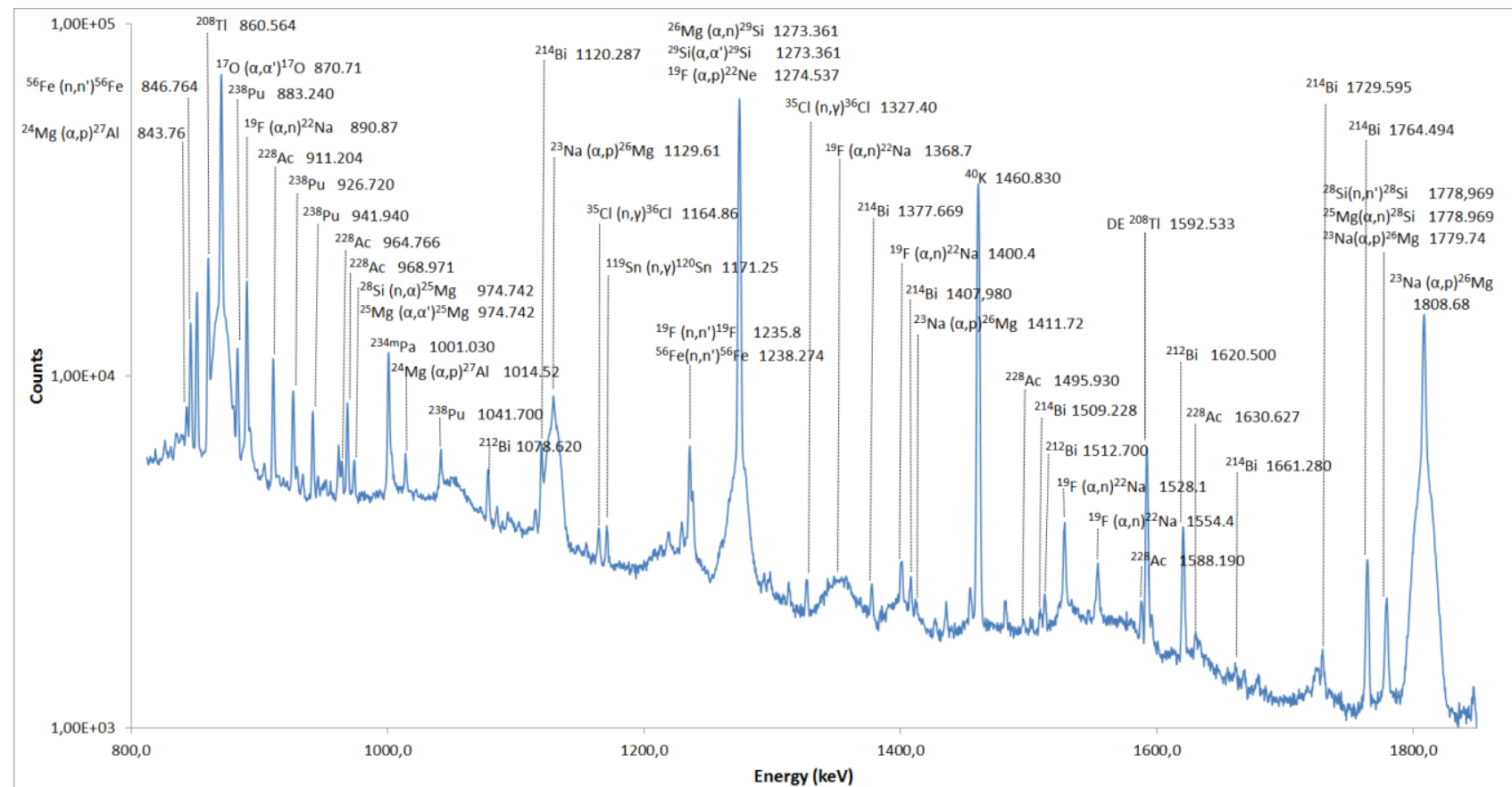

Figure 2: Gamma rays from natural radionuclides $\left({ }^{40} \mathrm{~K}\right.$, uranium and ${ }^{232} \mathrm{Th}$ decay chains $),(\alpha, \mathrm{x})$ and inelastic scattering reactions in the $800-1800 \mathrm{keV}$ energy range

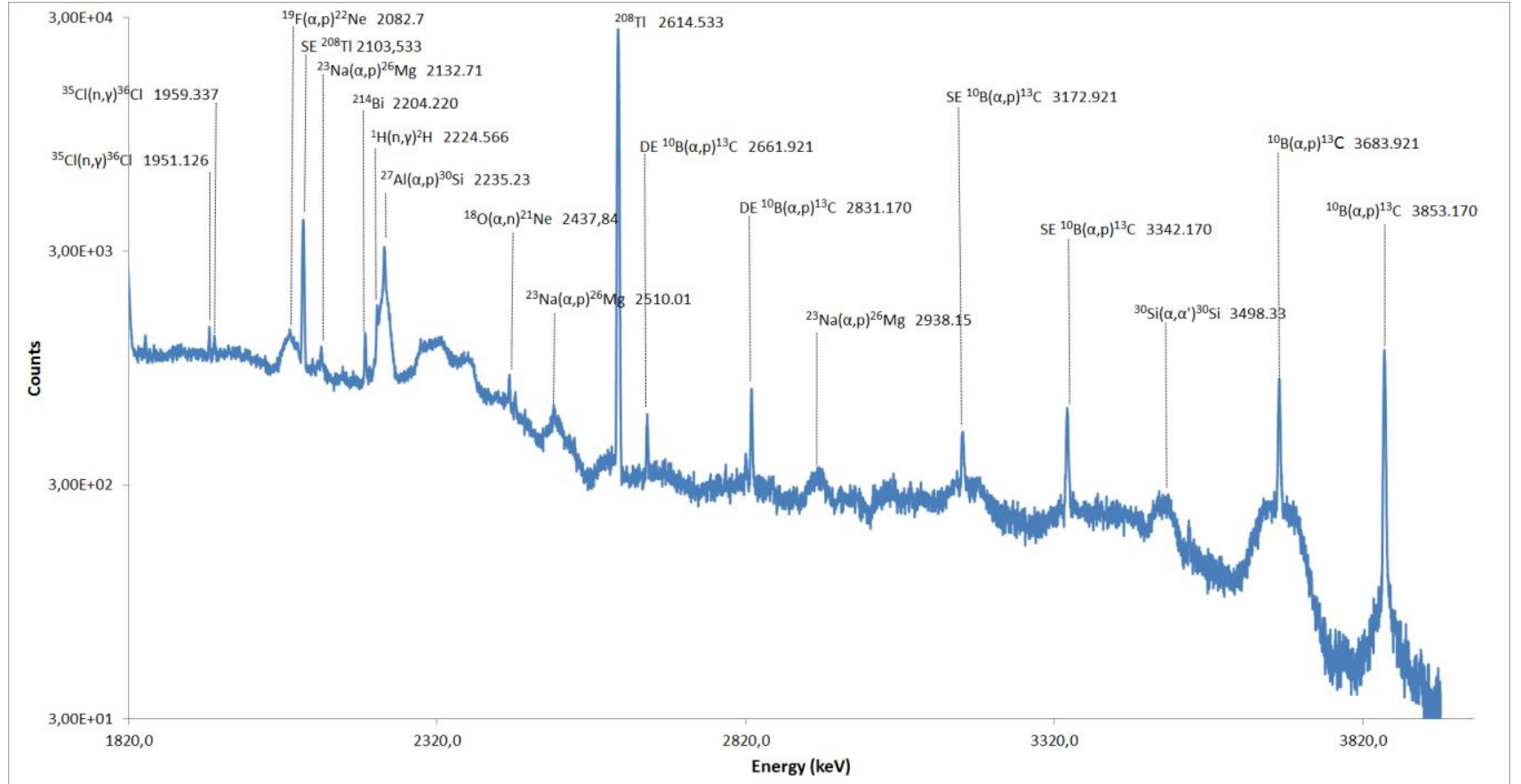

Figure 3: Gamma rays from natural radionuclides (uranium and ${ }^{232} \mathrm{Th}$ decay chains), $(\alpha, \mathrm{x})$ and inelastic scattering reactions in the $1800-4000 \mathrm{keV}$ energy range 


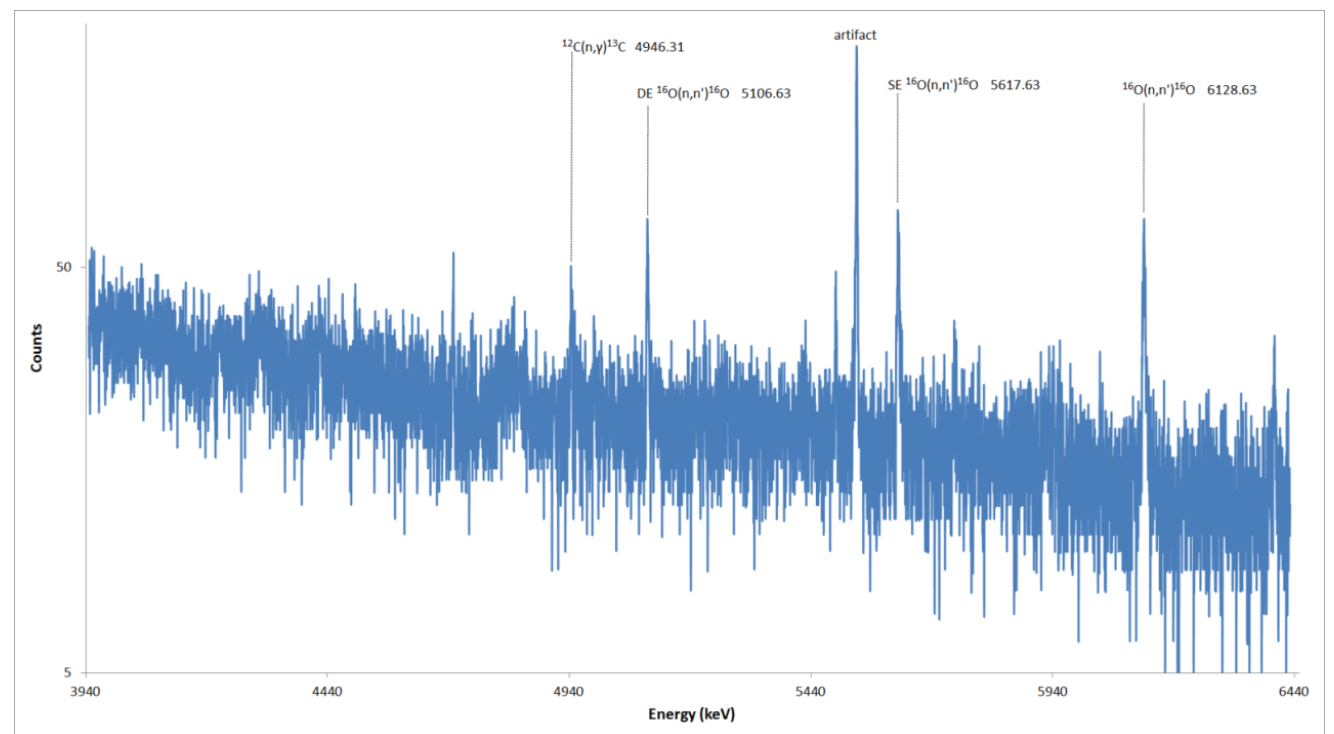

Figure 4: Gamma rays from neutron radiative capture and inelastic scattering reactions in the high-energy range (4000-6400 keV)

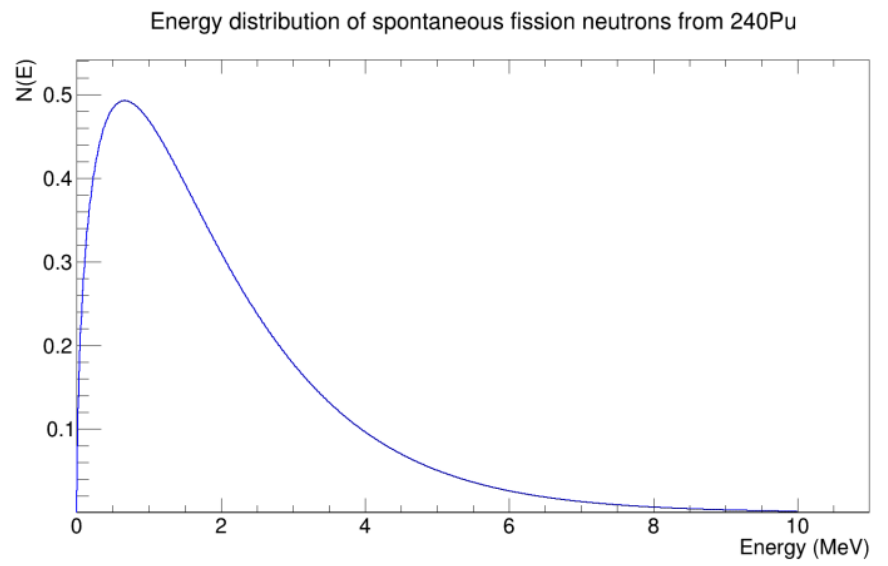

Figure 5: Prompt neutron spectrum from 240Pu spontaneous fissions, approximated as a Maxwell distribution N(E)

Table 1: Different high- and low-energy threshold combinations, and corresponding percentage of ${ }^{240} \mathrm{Pu}$ spontaneous fission neutrons between the two thresholds in the above Maxwell spectrum. In the two last columns, percentage below and above the low- and high-energy thresholds, respectively).

\begin{tabular}{|c|c|c|c|c|c|c|}
\hline \multicolumn{2}{|c|}{ Low-E threshold } & \multicolumn{2}{|c|}{ High-E threshold } & $\begin{array}{c}\text { \% of neutrons between } \\
\text { the low- and high-E } \\
\text { thresholds }\end{array}$ & $\begin{array}{c}\text { \% of neutrons } \\
\text { below the low-E } \\
\text { threshold }\end{array}$ & $\begin{array}{c}\text { \% of neutrons } \\
\text { above the high-E } \\
\text { threshold }\end{array}$ \\
\hline 60 & 426.9 & 800 & 3276 & 60.92 & 3.96 & 35.39 \\
\hline $\mathbf{6 0}$ & $\mathbf{4 2 6 . 9}$ & $\mathbf{1 0 0 0}$ & $\mathbf{3 7 9 8}$ & $\mathbf{6 8 . 9 5}$ & $\mathbf{3 . 9 6}$ & $\mathbf{2 7 . 3 6}$ \\
\hline 130 & 836.3 & 800 & 3276 & 53.32 & 11.29 & 35.39 \\
\hline 130 & 836.3 & 1000 & 3798 & 61.35 & 20.19 & 27.36 \\
\hline 210 & 1236 & 800 & 3276 & 44.42 & 20.19 & 35.39 \\
\hline 210 & 1236 & 1000 & 3798 & 52.45 & & 27.36 \\
\hline
\end{tabular}

Considering the high-energy threshold, the following settings are considered:

- a $800 \mathrm{keVee}$ threshold eliminates some gamma rays coming from $(\alpha, \mathrm{x})$ reactions and neutron inelastic scattering (as 1129 $\mathrm{keV}$ from ${ }^{26} \mathrm{Mg}, 1238$ from ${ }^{56} \mathrm{Fe}, 1274 \mathrm{keV}$ from ${ }^{29} \mathrm{Si}$ and ${ }^{22} \mathrm{Ne}$, $6128 \mathrm{keV}$ from ${ }^{16} \mathrm{O}$ ) but also neutron energy deposits above $3.276 \mathrm{MeV}$, i.e. $35 \%$ of spontaneous fission neutrons;
- a $1000 \mathrm{keVee}$ threshold would no more cut $1129 \mathrm{keV}$ gamma rays from ${ }^{26} \mathrm{Mg}$ (Compton Edge at $922 \mathrm{keV}$ ), but as it corresponds to a higher $3.798 \mathrm{MeV}$ neutron energy deposit, only $27 \%$ of spontaneous fission neutrons would be lost.

Different combinations of these low and high-energy thresholds are reported in

Table 1, along with the percentage of preserved or lost ${ }^{240} \mathrm{Pu}$ fission neutron losses. The 60 and $1000 \mathrm{keVee} \mathrm{low} \mathrm{and} \mathrm{high-}$ 
energy thresholds, respectively, seems to be a good trade-off because they cut the intense $59.54 \mathrm{keV}$ ray of ${ }^{241} \mathrm{Am}$ and a large part of high-energy gamma rays, while keeping more than $69 \%$ of spontaneous fission neutrons.

\section{CONCLUSION}

In order to propose plastic scintillators as a viable alternative to replace helium 3 gas counters in plutonium characterization coincidence collars, it is important to find mitigation means of their high sensitivity to gamma rays. These last, when emitted in correlation with other particles like in $(\alpha, n)$ and $(n, n$ ') reactions, or in gamma cascades, lead to parasitic coincidence that can be misidentified as coming from plutonium spontaneous fissions. The gamma spectrum of a $120 \mathrm{~L}$ waste drum filled with plutonium bearing glove box filters allowed us identifying, besides natural background rays $\left({ }^{40} \mathrm{~K}, \mathrm{U}\right.$ and $\mathrm{Th}$ chains) and well- known rays due to plutonium and americium radioactive decays, a number of less common gamma rays. These last are due to nuclear $(\alpha, x)$ reactions induced by the high alpha activity of $\mathrm{Pu}$ and $\mathrm{Am}$, as well as by neutrons from spontaneous fissions and $(\alpha, n)$ reactions. The analysis of the residual nuclei excited levels produced in alpha- and neutroninduced reactions allowed us identifying many such gamma rays, most of them with an energy larger than $1 \mathrm{MeV}$, which could lead to spurious coincidences in a measurement system with plastic scintillators. This analysis will lead us to use a high-energy rejection threshold to reject such gamma rays, while preserving the largest possible fraction of useful fission neutron pulses.

\section{REFERENCES}

[1] R.Kouzes, J.Ely, L. Erikson, W. Kernan, A. Lintereur, E. Siciliano, D. Stephens, D. Stromwold, R. Van Ginhoven, M. Woodring, "Neutron detection alternatives to ${ }^{3} \mathrm{He}$ for national security applications", Nuclear Instruments and Methods in Physics Research A 623 (2010) 1035-1045, January 2010.

[2] D.Henzlova, R. Kouzes, R.McElroy , P. Peerani, M. Aspinall, K. Baird, A. Bakel, M. Borella, M. Bourne, L. Bourva, F. Cave, R. Chandra, D. Chernikova, S. Croft, G. Dermody, A. Dougan, J. Ely, E. Fanchini, P. Finocchiaro, V. Gavron, M. Kureta et al. "Current Status of 3He Alternatives Technologies for Nuclear Safeguards" LA-UR-15-21201 Ver. 3, PNNL-24307, July 2015.

[3] B. Simony, C. Deyglun, B. Pérot, C. Carasco, N. Saurel, S Colas, J. Collot, Cross-talk characterization in passive neutron coincidence counting of radioactive waste drums with plastic scintillators, IEEE Transactions on Nuclear Science, Vol. 63, No. 3, June 2016, pp. 1513-1519.

[4] B. Simony, B. Pérot, C. Carasco, F. Jallu, N. Saurel, S. Colas, P. Girones, J. Collot, Passive neutron coincidence counting with plastic scintillators for the characterization of radioactive waste drums, IEEE Transactions on Nuclear Science, Vol. 64, No. 10 (2017) 2719-2724

[5] C. Carasco, B. Pérot, S. Normand, G. Sannié, "POLITRANI, A New Toolkit to Simulate Organic Scintillator Pulses", IEEE Transactions on Nuclear Science, Vol.61, No.4 , August 2014.

[6] G. Corre, K. Boudergui, G. Sannié, V. Kondrasovs, "Neutron Detection with Large Plastic Scintillators for RPM Applications", $20154^{\text {th }}$ International Conference on Advancements in Nuclear Instrumentation Measurements Methods and their Applications (ANIMMA), April 2015, Lisbon, Portugal. IEEE Engineers Inc, pp.7465625, 2015.
[7] S. P. Simakov, A. Pavlik, H. Vonach, S. Hlavac, "Status of experimental and evaluated discrete gamma-ray production at $E_{n}=14.5 \mathrm{MeV}$ ", INDC(CCP)-413, September 1998.

[8] G. F. Knoll "Radiation Detection and Measurement" $4^{\text {th }}$ ed, september 2010.

[9] S. A. Pozzi, J. A. Mullens, J. T. Mihalczo, "Analysis of neutron and photon detection position for the calibration of plastic ( $B C$ 420) and liquid (BC-501) scintillators", Nuclear Instruments and Methods in Physics Research A254 (2004) 92101.

[10] S. A. Pozzi, M. Flaska, A. Enqvist, I. Pazsit, "Monte Carlo and analatycal models of neutron detection with organic scintillation detectors", Nuclear Instruments and Methods in Physics Research A582 (2007) 629-637

[11] N. Ensslin, "Passive Nondestructive Assay of Nuclear material", NUREG/CR-5550, LA-UR-90-732, march 1991 